\title{
Mise à jour des dispositions du code de la santé publique relatives à la radioprotection
}

\author{
J.-L. GODET ${ }^{1}$, M.-L. PERRIN ${ }^{1}$
}

(Manuscrit reçu le 12 mai 2007, accepté le 9 juin 2007)

RÉSUMÉ Les dispositions du code de la santé publique concernant les rayonnements ionisants seront prochainement mises à jour pour achever la transposition de la directive 2003/122/Euratom du Conseil du 22 décembre 2003 relative au contrôle des sources radioactives scellées de haute activité et des sources orphelines et prendre en compte les nouvelles prérogatives accordées à l'Autorité de sûreté nucléaire (ASN). À cette occasion, des mesures de simplification administrative seront introduites dans le régime d'autorisation et de déclaration des sources de rayonnements ionisants et les contrôles réalisés par les organismes agréés par l'ASN seront renforcées.

ABSTRACT Updating of the French national regulation related to radiological protection.

The French national regulations, included in public health code, will be shortly updated to complete the transposition of the directive 2003/122/Euratom related to the control oh high activity sources and orphan sources and to take account of the new missions of the French Nuclear Safety Authority (ASN). This new context leads to simplify the existing authorisation and reporting system and to strengthen the task of approved body by ASN in charge of the control of facilities.

Keywords: radiological protection / regulation / sources / control

Le chapitre III « Rayonnements ionisants » (titre III, livre III) a été introduit dans le code de la santé publique dans le cadre de la codification des décrets 2002-460 du 4 avril 2002, 2003-270 du 24 mars 2003 et 2003-295 du 31 mars 2003 afin de transposer la directive 96/29/Euratom du conseil fixant les normes de base relatives à la protection sanitaire de la population et des travailleurs contre les rayonnements ionisants et la directive 97/43/Euratom relative à la protection sanitaire des personnes contre les dangers des rayonnements ionisants, lors d'expositions à des fins médicales.

\footnotetext{
1 Autorité de sûreté nucléaire, 6 place Bourgoin, 75012 Paris, France.
} 


\section{Une mise à jour nécessaire pour transposer la directive 2003/122/Euratom relative au contrôle des sources radioactives scellées de haute activité et des sources orpheline et prendre en compte les nouvelles prérogatives de l'Autorité de sûreté nucléaire}

Il s'avère désormais nécessaire de compléter ce chapitre afin, d'une part, de transposer la directive 2003/122/Euratom du Conseil du 22 décembre 2003 relative au contrôle des sources radioactives scellées de haute activité et des sources orphelines et, d'autre part, d'intégrer les nouvelles prérogatives de l'ASN introduites par la loi n ${ }^{\circ}$ 2006-686 du 13 juin 2006 relative à la transparence et à la sécurité en matière nucléaire. Cette occasion permet aussi de mettre à jour le chapitre III afin d'intégrer l'expérience acquise par l'ASN pour appliquer cette nouvelle réglementation. Les modifications liées à ce retour d'expérience ont pour objectif :

- d'introduire des mesures de simplification administrative, notamment en ce qui concerne le régime d'autorisation et de déclaration des sources de rayonnements ionisants (section III) ;

- de renforcer les mesures de contrôles prévues par le code de la santé publique ;

- d'apporter des précisions et des compléments dans la rédaction de plusieurs dispositions déjà en vigueur.

On notera que les arrêtés d'application prévus par le nouveau chapitre III seront pris après avis de l'ASN (consultation obligatoire prévue par l'article 4 de la loi du 13 juin 2006) et que plusieurs arrêtés d'application seront transformés en décision technique de l'ASN soumise à homologation du ministre chargé de la santé, conformément aux dispositions dudit article.

Le projet de décret reprend l'ensemble des modifications proposées par l'ASN dans son avis du $1^{\mathrm{er}}$ février 2007.

\section{Le réseau national de mesures de la radioactivité de l'environnement (Art. R.1333-11)}

Depuis avril 2002, a été publié l'arrêté DGSNR/DPPR du 17 octobre 2003 portant organisation d'un réseau national de mesures de la radioactivité de l'environnement (J.O. du 28 octobre) qui a été abrogé par l'arrêté du 27 juin 2005 (J.O. du 29 juillet 2005) pour dissocier le processus de transmission des résultats de mesures au réseau national et le processus d'agrément des laboratoires. Cependant, la nature et l'origine des données de mesures à transmettre au réseau national nécessitaient d'être explicitées. C'est l'objectif principal du nouvel article 
MISE À JOUR DES DISPOSITIONS DU CODE DE LA SANTÉ PUBLIQUE

R.1333-11 qui précise l'obligation de transmission au réseau national de toute mesure de radioactivité de l'environnement fixée par des dispositions législatives ou réglementaires et, par conséquent, réalisée par un laboratoire agréé ou par l'IRSN. Cela constitue un élargissement du cadre initial du réseau en ne le limitant pas exclusivement aux mesures liées aux impacts des rejets des installations nucléaires.

De plus, et pour tenir compte de la directive 2003/4/CE du 28 janvier 2003 concernant le droit d'accès du public à l'information en matière d'environnement, il est ajouté que toutes les mesures détenues par des collectivités territoriales, des services de l'État ou ses établissements publics (en dehors de leur qualité d'exploitant nucléaire ou d'organisme de recherche) doivent être transmises au réseau national si elles sont réalisées par des laboratoires agréés ou par l'IRSN. Les mesures de l'environnement de tout autre organisme, privé ou associatif, peuvent être transmises au réseau, avec l'accord de leurs détenteurs, sous réserve qu'elles proviennent de laboratoires agréés.

\section{Les laboratoires agréés pour la mesure de la radioactivité dans l'environnement (Art. R.1333-11-1)}

Les laboratoires seront maintenant agréés par l'ASN ; cette prérogative fait partie des décisions individuelles qui lui sont attribuées par la loi TSN (article 4). L'article R.1333-11 introduit cependant des précisions sur les délais de recevabilité (3 mois) et de réponse de l'autorité (ASN) aux dossiers de demande d'agrément présentés par les laboratoires (8 mois). Ce délai d'un an peut apparaître relativement long ; toutefois, cette durée intègre le temps nécessaire pour mettre en œuvre les essais d'intercomparaison organisés par l'IRSN et en traiter les résultats.

Une décision technique ASN homologuée sera nécessaire pour apporter les compléments nécessaires sur l'organisation du réseau de mesures et sur le régime d'agrément des laboratoires. À titre indicatif, il est par exemple prévu de maintenir le comité de pilotage du réseau introduit par l'arrêté du 28 juin 2005.

\section{Les effluents et les déchets produits dans les installations nucléaires de proximité (Art. R.1333-12)}

Cet article introduit un principe général de protection de la population (prise en compte du risque) applicable pour l'élimination des déchets et des effluents générés par les activités nucléaires de toute origine. Pour les INB, les ICPE autorisées et les installations minières, les règles particulières sont fixées par les réglementations qui leurs sont applicables. 
La nouvelle rédaction propose de réintégrer dans le domaine couvert par l'article R.1333-12 les installations classées soumises à déclaration, considérant que le champ de la simplification administrative prévue à l'article L.1333-4 est limité aux installations classées pour la protection de l'environnement soumises à autorisation. Par souci de cohérence, il apparaît souhaitable que les règles de gestion des effluents et sources générées par l'utilisation des sources radioactives non scellées, soumises à autorisation au titre du code de la santé publique, soient identiques, que la source en question soit soumise ou non à déclaration au titre des installations classées. Cette nouvelle rédaction permettra d'autoriser le rejet de certains radionucléides (y compris à vie longue) sans passer obligatoirement par une période de décroissance, sous réserve d'en évaluer l'impact. Une décision technique ASN homologuée apportera les précisions techniques nécessaires.

À titre de simplification administrative, la procédure d'approbation du projet de rejet et d'élimination des déchets et effluents sera liée à l'autorisation délivrée au titre de la section 3 afin de ne pas multiplier les procédures.

L'arrêté d'application de cet article n'a pas encore été publié. À défaut, l'autorité de contrôle applique l'arrêté du 30 octobre 1981 relatif aux conditions d'emploi des radioéléments artificiels utilisés en sources non scellées à des fins médicales ainsi que la circulaire DGS/DHOS du 7 juillet 2001 relative à la gestion des déchets et effluents susceptibles d'être contaminés par des radionucléides.

\section{La radioactivité naturelle renforcée dans les industries utilisant des matières premières contenant des radionucléides naturels (Art. R.1333-13)}

Depuis avril 2002, a été publié l'arrêté du 25 mai 2005 (J.O. n ${ }^{\circ} 126$ du $1^{\text {er }}$ juin 2005) définissant les catégories d'activités professionnelles faisant l'objet d'une surveillance des expositions aux rayonnements naturels. La préparation de cet arrêté d'application a mis en évidence la nécessité de certaines modifications, destinées à préciser les responsables des actions demandées et l'articulation avec le régime des installations classées. Plusieurs modifications sont apportées à cet article afin :

- d'intégrer les remarques de la commission européenne concernant la transposition du titre VII de la directive 96/29/Euratom du Conseil ;

- de préciser le responsable de la réalisation de l'étude (l'exploitant) ;

- de réviser les ministères signataires des arrêtés d'application ;

- de préciser le lien entre le code de la santé publique et la réglementation des installations classées. 
Le nouveau paragraphe ne rend pas nécessaire la réalisation d'une étude supplémentaire dès lors que l'étude d'impact exigée par la réglementation des installations classées inclut le risque radiologique. Il prévoit aussi, pour ces installations, la possibilité de prendre les mesures de radioprotection nécessaires par arrêté préfectoral dans le cadre de la réglementation «installations classées ».

La liste des installations concernées continuera à être établie et mise à jour par arrêté interministériel ; en revanche, les mesures techniques à prendre pour réduire les expositions seront fixées par décision de l'ASN, homologuée.

\section{La gestion des risques liés au radon dans les lieux ouverts au public (Art. R.1333-15, R.1333-15-1 et R.1333-16)}

L'arrêté du 22 juillet 2004 relatif aux modalités de gestion du risque lié au radon dans les lieux ouverts au public (J.O. du 11 août 2004) devra être modifié pour prendre en compte les prérogatives de l'ASN en ce qui concerne les décisions techniques mais la référence à un arrêté interministériel pour délimiter les zones prioritaires et les niveaux d'action est maintenue. Son domaine d'application est étendu aux modalités d'évaluation des performances des détecteurs utilisés.

L'expérience acquise par l'ASN en matière de gestion du risque lié au radon et lors de l'instruction des demandes d'agrément pour les organismes chargés des mesures de radon conduit à apporter plusieurs compléments à cet article. Ainsi, il est proposé :

- d'encadrer les programmes de formation des personnes chargées des mesures du radon exerçant au sein des organismes agréés par l'ASN ;

- des précisions sur la procédure d'agrément, en fixant un délai maximum de réponse de l'ASN (6 mois).

Les organismes seront agréés par l'ASN ; cette prérogative fait partie des décisions individuelles qui lui sont attribuées par la loi TSN (article 4). Une décision technique de l'ASN, homologuée, sera nécessaire pour apporter les compléments nécessaires sur le régime d'agrément. À titre indicatif, il est par exemple prévu de maintenir la Commission nationale d'agrément introduite par l'arrêté du 24 avril 2006.

Un alinéa supplémentaire a été introduit pour obliger les organismes agréés pour la mesure du radon à transmettre les résultats de leurs mesures à un organisme désigné à cet effet. La banque de données SISE Habitat, sous la responsabilité de la direction générale de la santé, en cours de constitution devrait permettre de regrouper ces données, utiles à l'évaluation de la politique de santé mise en place dans le cadre du Plan national santé environnement (PNSE). Un arrêté avec avis de la CNIL sera nécessaire pour préciser les modalités d'accès à ces informations. 


\section{Le régime général des autorisations et déclarations (Art. R.1333-17 à R.1333-43)}

La section 3 du chapitre III concernant le régime général des autorisations et déclaration est totalement réorganisée et complétée afin de :

- simplifier le régime des autorisations et déclaration mis en place en 2002, en intégrant les nouvelles prérogatives de l'ASN en matière de décision individuelle ;

- apporter des compléments nécessaires à la transposition de la directive 2003-122 ;

- apporter des précisions rédactionnelles pour améliorer la précision du texte.

L'élargissement souhaité du régime de déclaration, d'une part, le retrait de l'AFSSAPS en tant qu'autorité chargée de délivrer les autorisations pour les dispositifs médicaux et les radiopharmaceutiques, d'autre part, justifient cette nouvelle organisation. La nouvelle section 3 se veut plus simple d'accès.

Dans la $1^{\text {re }}$ sous-section, le premier article (R.1333-17) définit le domaine d'application de cette section 3 relative aux autorisations et aux déclarations. Il exclut celles qui sont couvertes par des régimes d'autorisation spécifiques (conformément à l'article L.1333-4) tels que celui applicables aux installations nucléaires de base ou aux installations classées pour la protection de l'environnement. Le second article (R.1333-18) précise les possibilités d'exemption au régime d'autorisation et de déclaration.

Les 4 sous-sections suivantes présentent les modalités du régime de déclaration (sous-section 2) et du régime d'autorisation (sous-section 3), ainsi que les dispositions communes à ces deux régimes (sous-section 4). Une nouvelle sous-section (sous-section 5) est réservée au cas particulier du transport de matières radioactives qui ne bénéficie pas (Art. L.1333-4) de la simplification prévue pour les INB, les ICPE et les activités minières.

Il est également précisé que les activités de distribution, d'importation ou d'exportation de sources radioactives, réalisées dans une installation classée pour la protection de l'environnement, restent soumises à autorisation de l'Autorité de sûreté nucléaire.

Les principales modifications apportées visent la réduction du champ des sources soumises à autorisation au profit d'un régime déclaratif, la suppression de la durée de validité des autorisations à 5 ans, en laissant la possibilité à l'ASN de fixer au cas par cas une telle limite si nécessaire mais qui ne devra pas excéder 10 ans et la possibilité de délivrer l'autorisation individuelle à une personne morale, et non plus seulement à une personne physique. 
Pour l'application de cette section, plusieurs décisions ASN homologuées seront nécessaires (R.1333-43), en particulier pour :

- exempter de l'autorisation ou de la déclaration certains appareils électriques bénéficiant, de par leur conception, d'une protection efficace ;

- établir la liste des installations soumises à déclaration ;

- préciser la liste des informations à joindre à la déclaration et à la demande d'autorisation ;

- indiquer les éléments sur lesquels peut porter l'autorisation délivrée par l'ASN ;

- fixer les conditions particulières d'emploi de certaines sources de rayonnements ionisants ;

- établir les règles techniques minimales de conception, d'exploitation et de maintenance des installations.

Le nouveau régime d'autorisation et de déclaration sera applicable dans un délai de 6 mois suivant la date de publication du décret, les dispositions soumises à des décisions de l'ASN étant applicables à compter de la date de publication des décisions homologuées ; les installations existant avant la date de publication, dûment déclarées et n'ayant pas fait l'objet de modification, sont dispensées de renouveler la déclaration prévue à l'article R.1333-22 du code de la santé publique.

\section{Le nouveau régime d'autorisation et de déclaration applicable au transport des matières radioactives (Art. R.1333-44)}

Un nouvel article est intégré afin de prévoir les modalités d'autorisation ou de déclaration des transports de matières radioactives. Un examen approfondi de la directive 96/29/Euratom, montre en effet que le transport des sources radioactives relève du champ couvert par cette directive et, de ce fait, oblige à prévoir une procédure d'autorisation ou de déclaration. À noter que la directive 2003/122/Euratom indique que la détention des sources radioactives de haute activité justifie une autorisation.

Dans ce contexte, il est proposé un régime d'autorisation ou de déclaration selon des critères techniques qui seront fixés par décision ASN ; ces critères techniques dépendent des catégories de colis transportés, celles-ci étant dépendantes de la réglementation technique internationale relative aux matières dangereuses introduite en France par voie d'arrêté (arrêté du $1^{\mathrm{er}}$ juin 2001 modifié relatif au transport des marchandises dangereuses par la route, dit « arrêté ADR »).

Du fait de ce contexte réglementaire spécifique aux transports des matières dangereuses, il est nécessaire de définir, par décision, une procédure d'autorisation et une procédure de déclaration spécifiques, distinctes des procédures générales décrites à la sous-section 3 . 


\section{Acquisition, distribution, importation, exportation, cession, reprise et élimination des sources radioactives (Art. R.1333-45 à R.1333-54)}

La modification de l'article R.1333-45 tient compte de l'introduction d'un régime déclaratif pour certaines sources radioactives. En pratique, lorsque le cumul des activités des sources détenues dépasse les seuils d'exemption, les dispositions de la section 4 s'appliquent à chaque source détenue. Il est, de plus, proposé d'exclure du système général de suivi et d'enregistrement les sources radioactives les opérations de transport de matières radioactives.

La cession ou l'acquisition de sources (Art. R.1333-46) n'est autorisée que pour les personnes qui justifient d'une autorisation ou d'un récépissé de déclaration (disposition nouvelle). Toutefois, cette obligation n'est pas nécessaire si l'activité de la source est inférieure aux seuils d'exemption.

Les responsabilités des fournisseurs (Art. R.1333-52) en matière de reprise des sources sont complétées en leur demandant notamment de disposer d'un site d'entreposage.

En s'appuyant sur l'article L.1333-7, les deux articles R.1333-53 et R.1333-54 prévoient que les fournisseurs de sources scellées doivent présenter des garanties financières dont les modalités du calcul sont fixées par voie réglementaire. Le montant de ces garanties sera fixé par arrêté, après avis de l'IRSN et de l'ANDRA. Ces dispositions répondent à une prescription de la directive 2003/122 qui introduit le principe des garanties financières. Un tel système existe déjà en France, en dehors de tout cadre juridique, sur la base du volontariat. Les fournisseurs français, regroupés au sein de l'association Ressources, ont établi un régime de caution permettant de faire face à une éventuelle défaillance d'un fournisseur.

L'article R.1333-54-1 a été complété pour tenir compte des dispositions de la directive 2003/122/Euratom concernant l'identification et le marquage des sources, qui pourront être introduites par voie de décision de l'ASN, homologuée. À noter que la publication de l'arrêté prévu à l'article R.1333-53 avait été différée, en attendant la publication de cette directive puis sa transposition en droit national. En attendant, les anciennes règles édictées par la CIREA ont continué à être appliquées (les dispositions transitoires introduites dans le décret 2002/460 permettent de prolonger les règles édictées par la CIREA, en attendant les textes d'application mentionnés au R.133-54-1). La publication de la décision ASN concernant l'identification et le marquage des sources scellées de haute activité ainsi que la nature des informations sur ces sources que le détenteur doit réunir, constitue le dernier texte de transposition de la directive 2003/122. 


\section{La radioprotection des patients (Art. R.1333-56 à R.1333-74)}

L'ancienne section 6 (protection des personnes exposées à des rayonnements ionisants à des fins médicales ou médico-légales) devient la section 5 ; depuis la publication du décret du 24 mars 2003 ont été publiés :

- l'arrêté du 17 juillet 2003 relatif à la mise hors service des appareils des radioscopies sans intensification d'image (J.O. du 21 août 2003);

- l'arrêté du 21 janvier 2004 définissant les éléments obligatoires d'information à transmettre aux patients après un acte de médecine nucléaire (J.O. du 6 février 2004) ;

- l'arrêté du 12 février 2004 définissant les niveaux de référence pour les examens radiologiques (J.O. du 16 mars 2004);

- l'arrêté du 18 mai 2004 relatif à la formation à la radioprotection des patients (J.O. du 19 juin 2004) ;

- l'arrêté du 19 novembre 2004 concernant les missions et la formation de la personne spécialisée en radiophysique médicale (J.O. du 28 novembre 2004) ;

- l'arrêté du 7 février 2005 fixant la liste des diplômes compatibles avec l'exercice des missions de la personne spécialisée en radiophysique médicale et permettant l'inscription à la formation spécialisée prévue à l'article 4 de l'arrêté du 19 novembre 2004 (J.O. du 24 février 2005);

- l'arrêté Santé du 26 septembre 2006 relatif aux informations dosimétriques devant figurer dans un compte rendu d'acte utilisant les rayonnements ionisants.

Cette section fait l'objet de quelques ajustements justifiés par l'expérience acquise pour son application. De plus, les arrêtés du ministre chargé de la santé sont remplacés par des décisions de l'ASN homologuées par le dit ministre, sauf celui concernant les missions de la personne spécialisée en radiophysique médicale (PSRPM). Les questions de responsabilité de ces spécialistes, traitées dans l'arrêté du 18 novembre 2004, justifient le maintien d'un arrêté.

\section{Les situations d'urgence radiologique et d'exposition durable aux rayonnements ionisants (Art. R.133375 à 95)}

L'ancienne section 7 (situations d'urgence radiologique et d'exposition durable aux rayonnements ionisants) devient la section 6 .

L'ancienne section 7 avait été complétée par décret n 2005-1179 du 13 septembre 2005 relatif aux situations d'urgence radiologique et portant modification du code de la santé publique (J.O. du 20 septembre 2005) afin d'achever la transposition de la directive 89/618/Euratom. 
Depuis la publication du décret du 24 mars 2003 ont été publiés :

- l'arrêté du 13 octobre 2003 définissant les niveaux d'intervention en situation d'urgence radiologique (J.O. du 4 novembre 2003) ;

- l'arrêté du 8 décembre 2005 relatif au contrôle d'aptitude médicale, à la surveillance radiologique et aux actions de formation ou d'information au bénéfice des personnels intervenants engagés dans la gestion d'une situation d'urgence (J.O. du 13 décembre 2005) ;

- l'arrêté du 4 novembre 2005 relatif à l'information des populations en situation d'urgence radiologique (J.O. du 22 novembre 2005).

Cette section ne fait, elle aussi, l'objet que de quelques ajustements. Les arrêtés d'application seront publiés après avis de l'ASN ; l'un d'entre eux est transformé en décision de l'ASN, homologuée.

Une nouvelle disposition (Art. R.1333-93) est introduite pour le contrôle des sources radioactives orphelines, du fait de la nécessité de transposer la directive 2003/122/Euratom. Pour ces sources, l'article R.1333-93 demande au préfet de déterminer les modalités de leur prise en charge, ainsi que les actions à mener en cas d'une telle découverte (transposition de la directive 2003/122).

\section{Contrôle technique des organismes agréés (Art. R.1333-95 à R.1333-97)}

L'ancienne section 5 «contrôle » devient la section 7. Elle contient des nouvelles dispositions sur le contrôle technique des organismes agréés, sur l'inspection de l'ASN et sur la déclaration des incidents en radioprotection.

L'article R.13333-95 provient de l'ex-article R.1333-43. Son déplacement est justifié afin de prévoir l'intervention de ces organismes pour toutes les activités nucléaires (INB, ICPE, ...), et non pas seulement à celles qui relèvent des autorisations et déclarations de la section 3 du code de la santé publique. Cet élargissement est cohérent avec les dispositions du code du travail qui prévoit l'intervention des mêmes organismes agréés pour toutes les activités nucléaires.

Du fait du rôle joué par les organismes agréés pour vérifier la conformité des installations contrôlées, il est proposé de créer les bases nécessaires à une déclaration des non-conformités (Art. R.1333-96). Un système d'alerte des autorités est également retenu dans le cas où les non-conformités constatées sont susceptibles d'entraîner une exposition importante des personnes (au-delà des limites réglementaires). 
L'article R.1333-97 prévoit une décision de l'ASN, homologuée, pour définir les modalités d'agréments des organismes de contrôle et les critères de nonconformité retenus pour l'alerte des autorités via les organismes agréés.

\section{Les inspecteurs de la radioprotection}

\section{(Art. R.1333-98 à R.1333-108)}

En collaboration avec l'ASN, la DPPR s'est engagée à simplifier la nomenclature des installations classées pour en améliorer la lisibilité (décret $n^{\circ} 2006-1454 \mathrm{du}$ 24 novembre 2006) mais aussi pour veiller à la cohérence avec le régime d'autorisation et de déclaration du code de la santé publique. Cependant, cette simplification pour l'utilisateur va se traduire par une perte d'information de l'ASN sur les dossiers instruits par les services en charge des installations classées. Les inspecteurs de la radioprotection restant compétent pour le contrôle de ces installations, des mesures réglementaires spécifiques sont donc à prévoir afin de garantir leur accès aux informations pertinentes à leur action (R.1333-99).

Les articles R.1333-99 à R.1333-108 reprennent les articles R.1333-94-1 et suivants introduits par le décret du 13 juin 2006, en les renumérotant. Les nouvelles prérogatives de l'ASN en matière de désignation des inspecteurs de la radioprotection (loi TSN) sont ainsi prises en compte.

\section{4. Évènements, incidents et accidents \\ (Art. R.1333-109 à R.1333-111)}

À la lumière des derniers incidents survenus en radiothérapie (Lyon, Grenoble, Épinal), il apparaît nécessaire d'apporter des précisions sur le régime de déclaration prévu au L.1333-3 ; une décision ASN sera nécessaire pour ensuite préciser la nature des évènements significatifs soumis à déclaration (R.1333-111).

\section{Homologation des décisions techniques de l'Autorité de sûreté nucléaire}

La section 8, fixant les modalités d'homologation des décisions techniques à caractère réglementaire de l'ASN, est ajoutée.

\section{Annexes 13-7 et 13-8 du code de la santé publique}

\section{Annexe 13-7 - Définition et terme utilisés}

Cette annexe est modifiée afin d'intégrer les nouveaux termes introduits par les dispositions relatives aux sources de hautes activités (« défaillant», «fournisseur », 
« sources radioactives orpheline » et « source radioactive de haute activité »). Un complément est ajouté pour la définition de la dose équivalente engagée afin de la rendre cohérente avec celle donnée par la directive 96/29/Euratom.

\section{Annexe 13-8 - Seuils d'exemption}

La nouvelle annexe 13-8 reprend l'ancienne annexe 13-8 en y ajoutant, d'une part, la liste complémentaire des radionucléides introduits par l'arrêté du 2 décembre 2003 définissant les seuils d'exemption pour les paramètres autres que ceux figurant à l'article R.1333-27 du CSP (J.O. du 6 janvier 2004) et, d'autre part, les seuils définissant les sources de haute activité fixés par la directive 2003/122/Euratom. 